\section{Diagnosis of brain death}

SIR,-I find myself in a strange position with regard to the current brain death debate. I am a doctor and also chairman of the Association of Community Health Councils. From where I am I think that I really can see both sides.

I would like to suggest that the BMA (or the colleges) approach the BBC and apply to do an Open Door programme under its community access scheme. No doubt the application could be backed up by the suggestion that the programme could be made available to medical schools, postgraduate centres, and patient and community groups as part of continuing education about transplants. This basis for action would avoid the problems of editorial freedom and allow the profession to produce its version of a balanced programme. I would obviously press the case for the involvement of patients and community interests in the making of the programme.

Of course, these programmes are not transmitted at peak times but at least the resources of the BBC would be seen to be available. It might even be that some part of the BBC current affairs machine chose to feature parts of the programme at a peak time-subject, of course, to editorial judgments, news value, and so on.

Editorial freedom is one of those things, like clinical freedom, that we all have to be prepared to die for; the pity at the moment is that it is only those with chronic renal failure who are having actually to make the sacrifice. I offer my admittedly naive suggestion as a way in which both the profession and the media could work together and climb up out of this mess rather than wait for either to climb down.

\section{R K GRIFFITHS}

Health Services Research Centre, University Department of Social Medicine

Birmingham B15 2T

\section{Toxic shock and tampons}

SIR,-We wish to draw attention to a case of toxic shock syndrome in Auckland, New Zealand, with reference to your leading article (1 November, $\mathrm{p}$ 1161)

On 22 July 1980 a 21-year-old New Zealand woman was admitted to hospital at the end of a normal period, during which she uninterruptedly used a brand of tampons bought and manufactured in New Zealand. She was extremely unwell with a four-day history of diarrhoea and one to two days of vomiting, rigors, myalgia, and sore throat. She had facial oedema, conjunctivitis, a widespread diffuse erythematous rash, and pharyngitis. She was shocked and her blood pressure was $75 / 40 \mathrm{~mm} \mathrm{Hg}$. Vaginal examination was normal apart from a slight vaginal discharge and there was no evidence of pelvic inflammatory disease. Penicillin-resistant Staphylococcus aureus was cultured from the vaginal discharge but no pathogens were cultured from other specimens including blood cultures. She was resuscitated, given intravenous antibiotics, and recovered. Desquamation of the rash began on the fifth hospital day and continued for two weeks. Staph aureus was not cultured from her vagina after antibiotic therapy finished.

Two months later she was readmitted with a similar though less severe illness at the end of a period, during which she had used the same brand of tampons. This time she had a profuse vaginal discharge but again a striking absence of objective signs of pelvic inflammatory disease. Staph aureus was again cultured from the discharge but attempts to culture it from elsewhere in her body were not successful. She took cloxacillin and recovered, and the organism was eliminated. She has remained well. Staph aureus was not cultured from the unused boxed tampons.

Toxic shock syndrome, as might be expected, is not seen only in the United States, and tampons manufactured elsewhere may thus be causally associated. This woman had a recurrence despite initial elimination of vaginal Staph aureus. Despite widespread medical and lay publicity in New Zealand generated by this patient, we are unaware of others. This case is being reported in detail in the New Zealand Medical Fournal.

S IEA

Auckland Hospital,
Auckland 1, New Zealand

R B ElLIS-PEGLER

SIR,-We were surprised to read in your leading article "Toxic shock and tampons" (1 November, $\mathrm{p} \mathrm{1161)}$ that "A significant association has been shown between the use of tampons .... and the development of the toxic shock syndrome. In one study Staph aureus was isolated from 43 of 44 vaginal cultures taken from women with the toxic shock syndrome before antimicrobial treatment was started, whereas it was isolated from the vagina of only four of 55 control women. ..." While this suggests an association between Staph aureus and the syndrome it neither examines nor provides evidence regarding the role of tampons.

One of us chanced to be in the United States recently when this syndrome was the subject of massive publicity. As there are some 80 million menstruating women in the United States, $70 \%$ of whom it is estimated use tampons, this publicity seemed a contribution to the creation of disproportionate anxiety, ill health, and probably futile activity.

JAMES MCCORMICK ShaNe AllWRIGHT University Department of Community Health, Dublin 2

* * Our leading article stated that " $95 \%$ of all reported cases of toxic shock syndrome in women have occurred during or soon after a menstrual period" and analysis showed a substantial excess of users of one particular brand of tampon. How much plainer could an association be ?-ED, $B M \mathcal{F}$.

Staphylococcal septicaemia after insertion of an intrauterine contraceptive device

SIR,-Your recent leading article on toxic shock and tampons (1 November, $p$ 1161) prompts me to report a case of pelvic infection and staphylococcal septicaemia eight days after the insertion of a Lippes Loop. Pelvic infection is a recognised complication of intrauterine contraceptive devices (IUCD) ${ }^{\mathbf{1}}$; but although there have been two reports of endocarditis occurring in susceptible patients following the insertion of an IUCD ${ }^{23}$ septicaemia is rare.

A previously healthy 31-year-old married woman had a Lippes Loop IUCD fitted at a family planning clinic. Three days later she developed sweating, vomiting, confusion, and cough and during the following 48 hours became disorientated with hallucinations. She was referred to hospital with suspected encephalitis and on admission was febrile $\left(38.8^{\circ} \mathrm{C}\right)$ and stuporose but responded to simple commands. Blood pressure was $95 / 60 \mathrm{~mm}$ $\mathrm{Hg}$ but there were no other abnormal signs. Haemoglobin was $12.2 \mathrm{~g} / \mathrm{dl}$, white blood count $4.0 \times 10^{9} / 1$ ( $80 \%$ neutrophils), erythrocyte sedimentation rate $70 \mathrm{~mm}$ in the first hour; cerebrospinal fluid normal. Chest $x$-ray examination revealed patchy consolidation in the upper lobes of both lungs and an electroencephalogram showed bilateral non-specific abnormality.

Three blood cultures taken on admission yielded penicillin-resistant Staphylococcus aureus. She was treated with high-dose intravenous cloxacillin and 24 hours after starting the antibiotic had improved markedly and the IUCD was removed. Culture from the coil and also from a high vaginal swab yielded Staph aureus with a similar antibiogram to that of the organism cultured from the blood. Subsequent recovery was uneventful, although repeat chest $x$-ray examination showed small abscess cavities in the upper lobes of both lungs. The patient was discharged four weeks after admission and serial chest radiographs have confirmed complete resolution of the pneumonia and abscesses.

There is little doubt that this patient's staphylococcal septicaemia with lung abscess formation and encephalopathy originated in the genital tract. The patient was both toxic and shocked but was different from patients with the recently described toxic shock syndrome in that her blood culture was positive for Staph aureus. The case provides another example of the importance of this organism as a cause of infection associated with the insertion of foreign bodies into or through the vagina.

A M GedDES

\section{Department of Communicable and Tropical Diseases,}

Birmingham B9 5ST

1 Anonymous. Br Med f 1976;ii:717-8.

3 de Swiet. M, Ramsay ID, Rees GM. Br Med f 1975;iii:76-7.

\section{Fulminant Streptococcus pyogenes}

\section{infection}

SIR,-I read with interest your "Lesson of the Week" (22 November, p 1412). We had a patient in February 1978, who did not present a classical picture.

A 46-year-old woman was admitted to the surgical ward with severe intractable pain in her right hip. She had had a "flu-like" illness with a sore throat for one week, as had her daughter. She had received no antibiotics. The pain had become progressively more severe over the previous 24 hours. She was dehydrated and confused with a pyrexia of $37 \cdot 6^{\circ} \mathrm{C}$. There was no history of trauma but she had chronic lumbar disc disease. Over the next few hours she developed an area of bruising, oedema, and skin desquamation over her right hip and buttock; this spread rapidly over her thigh. It was not a true cellulitis, just a raw bleeding area where the skin had sloughed off. The area was very cold to touch. She had three sets of blood cultures and throat and skin swabs taken. Benzylpenicillin, 5MU six hourly, and gentamicin were started intravenously, as necrotising fasciitis was suspected. She became shocked 24 hours after admission (10 hours after starting antibiotics) and was resuscitated with plasma. Twelve hours later the lesions had spread down to her right knee, extending as far as her lumbar region and across the midline. She then had a cardiac arrest and all attempts to resuscitate her failed.

Streptococcus pyogenes ( $\beta$-haemolytic streptococcus, Lancefield group A) was later isolated from her blood cultures and throat and skin swabs. At necropsy she had a grossly oedematous 